\title{
Severe septic arthritis of the temporomandibular joint with pyogenic orofacial infections: A case report and review of the literature
}

\author{
DI XIAO, XUEFENG FENG, HAITAO HUANG and HUIXIN QUAN
}

\author{
Department of Stomatology, The First Affiliated Hospital of Dalian Medical University, Dalian, Liaoning 116023, P.R. China
}

Received March 31, 2016; Accepted March 3, 2017

DOI: $10.3892 /$ etm.2017.4510

\begin{abstract}
Septic arthritis of the temporomandibular joint (SATMJ) is a rare entity that has only been reported a few dozen times worldwide. However, SATMJ is an acute infectious disease and associated with a high risk for misdiagnosis, which results in severe complications for patients. The present study reported a case of an 83-year-old female patient with severe pyogenic orofacial infections caused by right-side SATMJ, which was not cured by abscess incision drainage and anti-microbial treatment. Finally, the clinical signs were resolved by condylectomy and debridement of the erosive septic TMJ, after which the function of the TMJ was completely restored. In conclusion, a timely and correct diagnosis with early treatment is critical to preventing acute complications and late sequelae of SATMJ. In addition, literature associated with this topic was reviewed and discussed.
\end{abstract}

\section{Introduction}

Septic arthritis of the temporomandibular joint (SATMJ) is a rarely reported disease characterized by pain, fever, swelling and even loss of TMJ function. In the past decades, only a few dozen cases of SATMJ have been reported; however, an increasing number of cases of SATMJ in children and adults have been reported in recent years (1-4). The disease predominantly occurs in male adults, the mortality rate of SATMJ is as high as $12 \%$, and up to $75 \%$ of survivors develop significant functional disability in the involved joints (5). The etiologies of SATMJ include general and local predisposing factors. Certain systemic and autoimmune diseases may cause septic arthritis, such as rheumatoid arthritis, diabetes, systemic lupus erythematous and hypogammaglobulinemia (6-8). On the other hand, direct extension of head and neck infection,

Correspondence to: Dr Huixin Quan, Department of Stomatology, The First Affiliated Hospital of Dalian Medical University, 222 Zhongshan Road, Xigang, Dalian, Liaoning 116023, P.R. China E-mail: huixinquan@hotmail.com

Key words: septic arthritis, temporomandibular joint, pyogenic orofacial infections, condylectomy, treatment local trauma and burn wounds affecting the TMJ obviously increase the morbidity of SATMJ (9-12). Studies have reported that iatrogenic causes, such as third molar abstraction and distraction osteogenesis, also lead to SATMJ $(13,14)$. On a microbiological level, the pathogenesis of SATMJ involves hematogenous dissemination of causative microorganisms or direct extension of a contiguous infection (15), and the infection was often caused by bacteria including Staphylococcus aureus, Neisseria gonorrhea, Streptococcus and Aspergillus flavus (6,16-19). Although no criteria have been established on the diagnosis and no consensus has been reached on the management of SATMJ, most studies employed aspiration and analysis of TMJ fluid, blood chemistry, imaging and clinical examination are for its diagnosis and treatment. Failure to promptly treat SATMJ may result in irreversible damage of the TMJ structure, leading to long-term complications that result in impaired joint mobility and function, including bone deformity, fibrosis and ankyloses $(15,17,20,21)$.

The present study reported a case of acute and severe SATMJ with pyogenic orofacial infections, which was not improved after abscess incision drainage and antibiotic treatment. Finally, the clinical signs were resolved by condylectomy and debridement of the erosive septic TMJ, which completely restored the function of the TMJ. A review of the literature associated with this topic was also performed.

\section{Case presentation}

History. A non-immunocompromised 83-year-old woman presented at the Department of Stomatology of the First Affiliated Hospital of Dalian Medical University (Dalian, China) on October 14th 2014, with a chief complaint of pain and swelling in the right buccal, temporal, parapharyngeal and pre-auricular regions for twenty days, including increasing difficulty opening her mouth. She had smoked for 60 years and was still smoking on admission, but was otherwise generally healthy. The patient reported that she initially had a pustule with a slight swelling in the skin of the inside of the right cheek; she then sought medical attention and was treated with antibiotics at another hospital. However, ten days later, the swelling subsequently aggravated and radiated to the right temporal and pre-auricular region, alongside which she presented with progressing dysphagia. Prior to the onset of the 
disease, the patient did not feel any spontaneous pain in the tooth or residual roots.

Clinical examination. The physical examination revealed a severe, ill-defined swelling with tenderness in the right-side orofacial region, mainly centering over the right buccal, pre-auricular and temporal regions, which was only mildly warm and erythematous, and which limited the patient's ability to open her mouth to $10-15 \mathrm{~mm}$ (Fig. 1). A fistula with pyorrhea was observed in the right cheek. Upon opening of the mouth, a tumescent palatoglossal arch was also found. Pitting edema were distributed over the patient's bilateral lower legs. The patient's vital signs were as follows: Body temperature, $37.1^{\circ} \mathrm{C}$; heart rate, 88 beats per minute; blood pressure, $140 / 80 \mathrm{mmHg}$; and oxygen saturation, $98 \%$ in room air. A blood culture was obtained. Hematological values were as follows: White blood cell count, 7,370/ $\mu \mathrm{l}$; neutrophils, 5,520/ $\mu \mathrm{l}$; hemoglobin, $98 \mathrm{~g} / \mathrm{dl}$; platelet count, 399,000/ $\mu \mathrm{l}$; albumin, $31.5 \mathrm{~g} / \mathrm{l}$; globulin, $37.9 \mathrm{~g} / \mathrm{l}$; and a reverse albumin/globulin ratio. All other laboratory values were within normal limits. In order to confirm the diagnosis, further investigation was pursued. Computed tomography (CT) scans of the craniofacial region revealed a destructive soft tissue mass, involving the articular eminence and condylar head. In addition, abscesses were seen in the right buccal and temporal spaces as well as parapharyngeal spaces (Fig. 2). The CT scans and a panoramic radiograph showed that the condylar head was diffusely radiolucent in the middle (Figs. 2-4).

Treatment. Based on the patient's history as well as the clinical and radiographic examination, a presumptive diagnosis of pyogenic orofacial infections was made. Thus, the patient was admitted to the hospital and adequate drainage of the abscess was performed under local anesthesia via two incisions at buccal (by enlarging the fistula) and temporal regions. During the surgery, $\sim 50$ milliliters of purulent material was withdrawn from the abscess and sent for culture and antibiotic sensitivity testing, and another 5 milliliters of pus was aspirated from the glossopharyngeal space with a long needle (Fig. 1B and C). The patient reported immediate improvement of her symptoms. After evacuation of pus, repeated irrigation with saline was performed each day through the percutaneous catheters and empirical intravenous antibiotic therapy was commenced (cefazolin sodium, $2 \mathrm{~g} / 12 \mathrm{~h}$; metronidazole, $0.5 \mathrm{~g} /$ day). Analgesics were also given to reduce the patient's pain. To correct hypoproteinemia, the patient was treated with intravenous human serum albumin and fresh frozen plasma. On the 7th day after the abscess incision, purulent material culture and antibiotic sensitivity testing showed positivity for Escherichia coli, which was sensitive to imipenem and meropenem. According to the test results, the patient was continually treated with intravenous antibiotics, which was switched to imipenem $(1 \mathrm{~g} / 12 \mathrm{~h})$. Although the swelling of the right facial region decreased and pain was relieved after a 25-day treatment, pyorrhea did not appear to improve. As the symptoms had not resolved, another CT scan was performed, which revealed that an abscess remained in the anterior region of the right-side TMJ, suggesting that exploration of the joint was necessary. After the patient gave her informed consent, a right mandibular condylectomy and debridement via a pre-auricular approach was performed to excise the lesion of the right-side

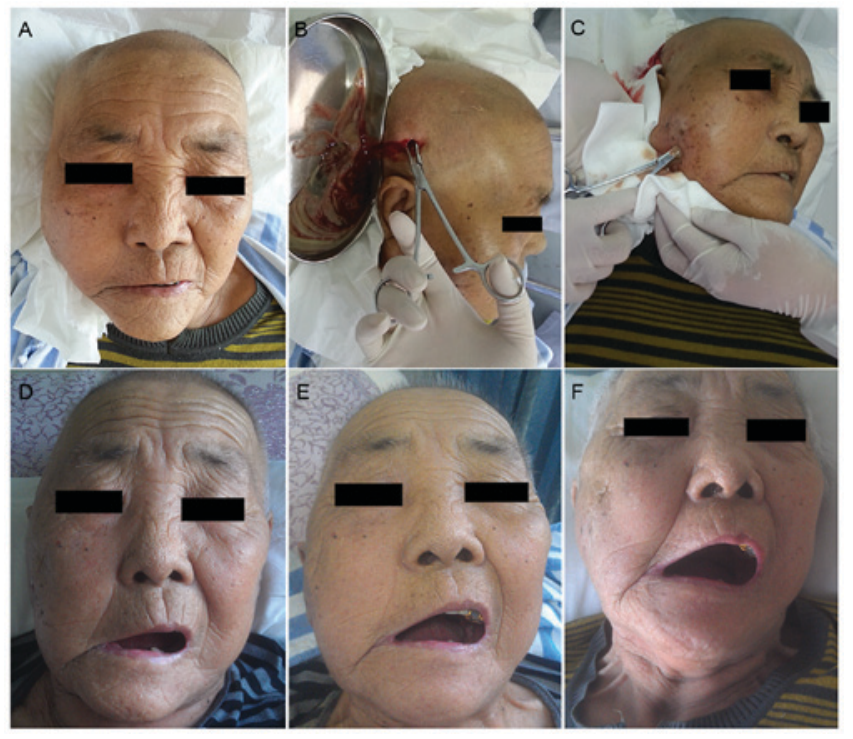

Figure 1. Clinical manifestations and abscess incision drainage. (A) A severe, ill-defined swelling with tenderness in the right-side orofacial region, mainly centering over the right buccal, pre-auricular and temporal regions. (B and C) Adequate drainage of the abscess was performed under local anesthesia via two incisions at the buccal and temporal regions. Large amounts of purulent material were withdrawn from the abscess and subjected to culture and antibiotic sensitivity testing. (D-F): Mouth-opening of the patient at different time-points: (D) Prior to condylectomy, (E) at 1 month after condylectomy and $(\mathrm{F})$ at 4 months after condylectomy.

mandibular condyle under general anesthesia. During the surgery, a large amount of pus was drained from the abscess and a right mandibular condyle with bone erosion was seen (Fig. 5). After the condylectomy, a vertical defect was observed but not repaired, as the patient was too old to tolerate a bigger operative trauma and a longer general anesthesia. The wound was lightly packed with an iodoform gauze used as a drainage, which was gradually taken out over 1 week, and the wound was later closed in layers. The tissue that filled the joint space was sent for histopathological examination, which identified inflammatory granulation tissue with patchy infiltrates of lymphocytes (Fig. 5E and F). Therefore, the final diagnosis was right-side SATMJ. At 2 months after condylectomy, a CT scan revealed improvement of the abscess around the right-side TMJ region (Fig. 2) and after another 2 months, her mouth-opening range reached $\sim 35 \mathrm{~mm}$ without any right face pain (Fig. 1F). After 1 year of post-operative follow-up, neither recurrence nor complications were observed. Unfortunately, the superior buccal branches of the facial nerve (VII) were unwittingly damaged during the course of treatment. While it is difficult to state when this nerve damage occurred, it was likely to have occurred during the process of abscess incision and draining, although pseudo facioplegia or condylectomy may have also caused damage. This damage was permanent, the symptoms were still present 1 year following treatment and although the damage did not affect normal chewing and eating, the patient drooled when liquid was gargled.

\section{Discussion}

Compared with hips and knees, TMJs are affected by septic arthritis much less frequently $(6,22,23)$. Usually, trauma, head 


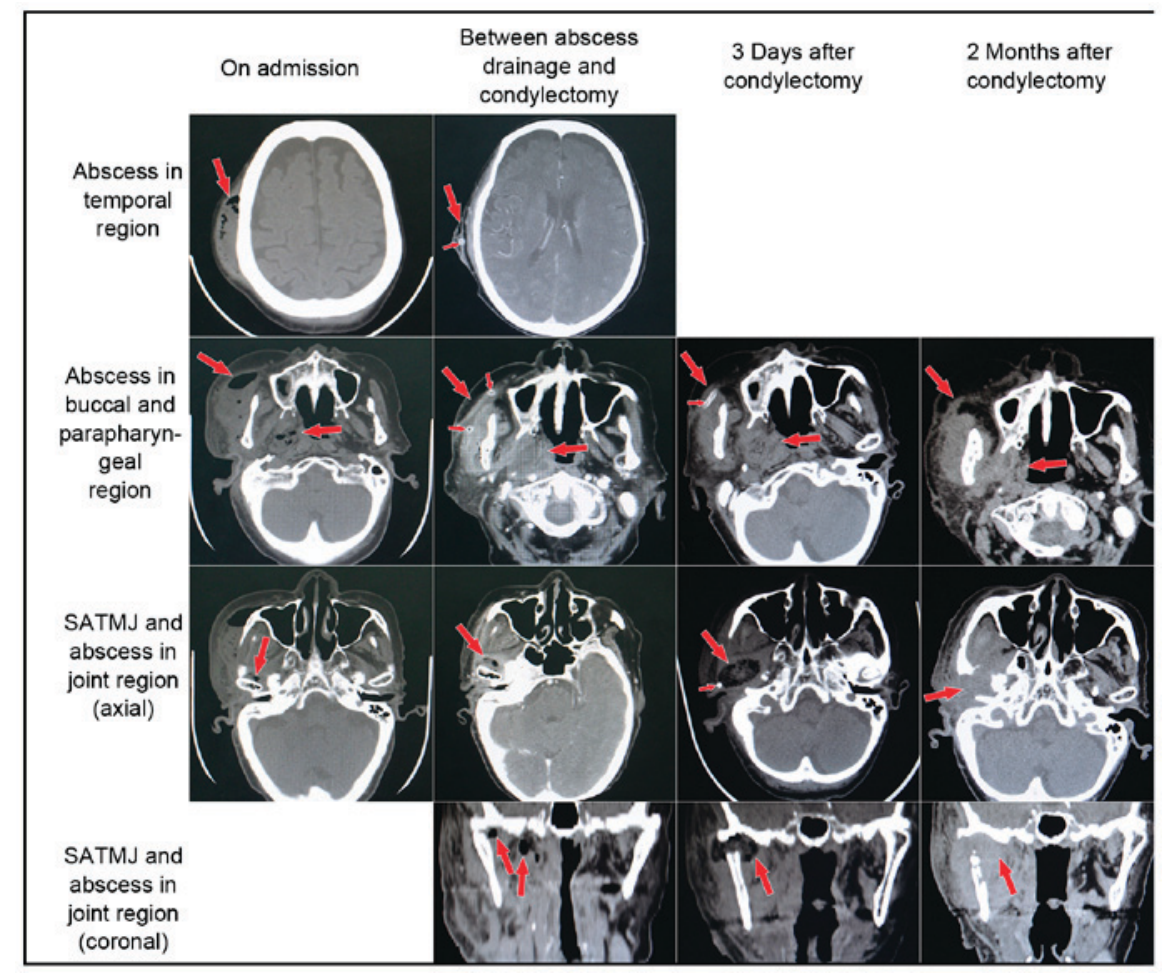

Figure 2. Computed tomography scans of the craniofacial region at different time-points (axial and coronal scans). When the patient was admitted to the hospital, abscesses were detected in the right buccal and temporal spaces, as well as the parapharyngeal space, and the condylar head was diffusely radiolucent in the middle. Between the abscess drainage and the condylectomy, an abscess remained in the anterior region of the right-side TMJ and the condylar head was diffusely radiolucent in the middle. After the condylectomy, the abscess gradually disappeared and was replaced with granulation tissues in the joint space. The bigger arrows show continuous variable processes of the abscesses and destruction of the right-side TMJ. The smaller arrows show the percutaneous catheters. The images in the bottom panel were magnified x1.4 to improve clarity. SAMTJ, septic arthritis of the temporomandibular joint.

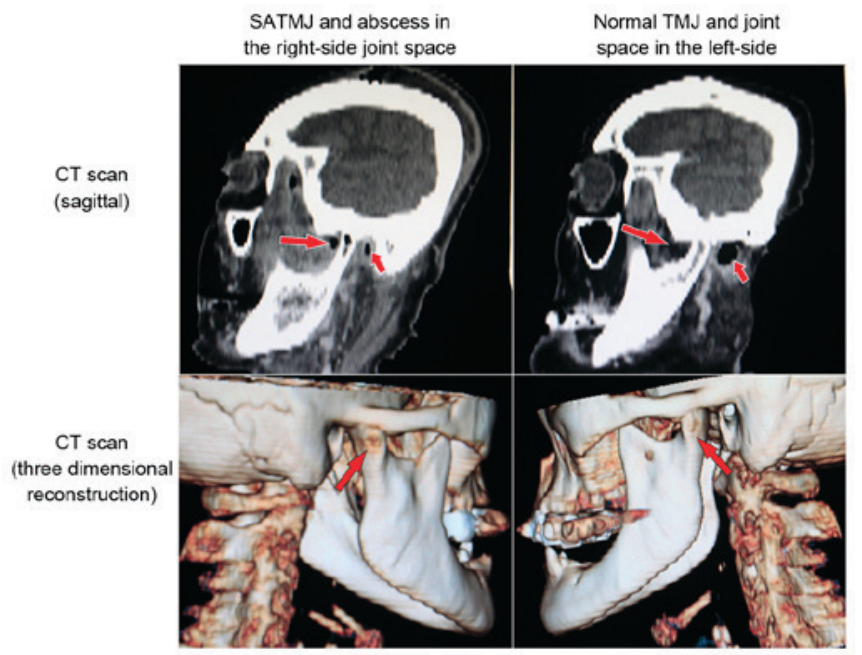

Figure 3. Comparison of CT scans (sagittal scans and 3D reconstruction) between SATMJ on the right side and normal TMJ on the left side. In the sagittal scans, the bigger arrows show that right-side TMJ was diffusely radiolucent in the middle, an abscess in the joint space was seen and the left-side TMJ was normal. The smaller arrows show the external auditory canal. In the $3 \mathrm{D}$ reconstruction, the bigger arrows show destruction of the right-side TMJ and a normal TMJ on the left side. SAMTJ, septic arthritis of the temporomandibular joint; CT, computed tomography.

and neck infection or TMJ arthrosis are the major causes of SATMJ, while it may also arise secondary to mastoiditis and otitis media (13). It is generally accepted that occurrence of SATMJ often occurs due to either local spread or hematogenous dissemination from a distant primary infection $(5,6,8,15)$. In addition, the TMJ synovium is highly vascular and has no limiting basement membrane. Consequently, acute or blunt trauma with resulting capsular injury is particularly likely to bring the infection into the joint space via these two routes $(13,24)$. As mentioned above, in the present case, SATMJ appeared to originate from pyogenic orofacial infections invading the TMJ. However, treatment by abscess incision and drainage as well as intravenous antibiotic therapy was not sufficient. It was therefore assumed that the occlusal trauma and mechanical stress resulting from edentulous jaws of this patient had damnified the aging mandibular condyle, which initiated arthritic changes, including destruction and necrosis in the TMJ, and created an environment supportive of bacterial infection. Subsequently, abscess spreading from the joint space to adjacent spaces caused the severely pyogenic orofacial infections (25). In other words, the present case suggested that SATMJ itself may be an independent bacterial disease and a source of infections to peripheral tissues, which is distinct from aseptic inflammations similarly caused by occlusal trauma, such as osteoarthritis of TMJ.

Misdiagnosis of SATMJ causes delays in its cure as well as irreversible complications, which may even be life threatening. Therefore, timely and correct diagnosis with early treatment is critical to preventing acute complications and late sequelae. Despite the lack of unified diagnostic criteria for SATMJ, numerous studies have consistently described the determination of clinical manifestations, imaging findings, joint aspiration and joint fluid analysis and 


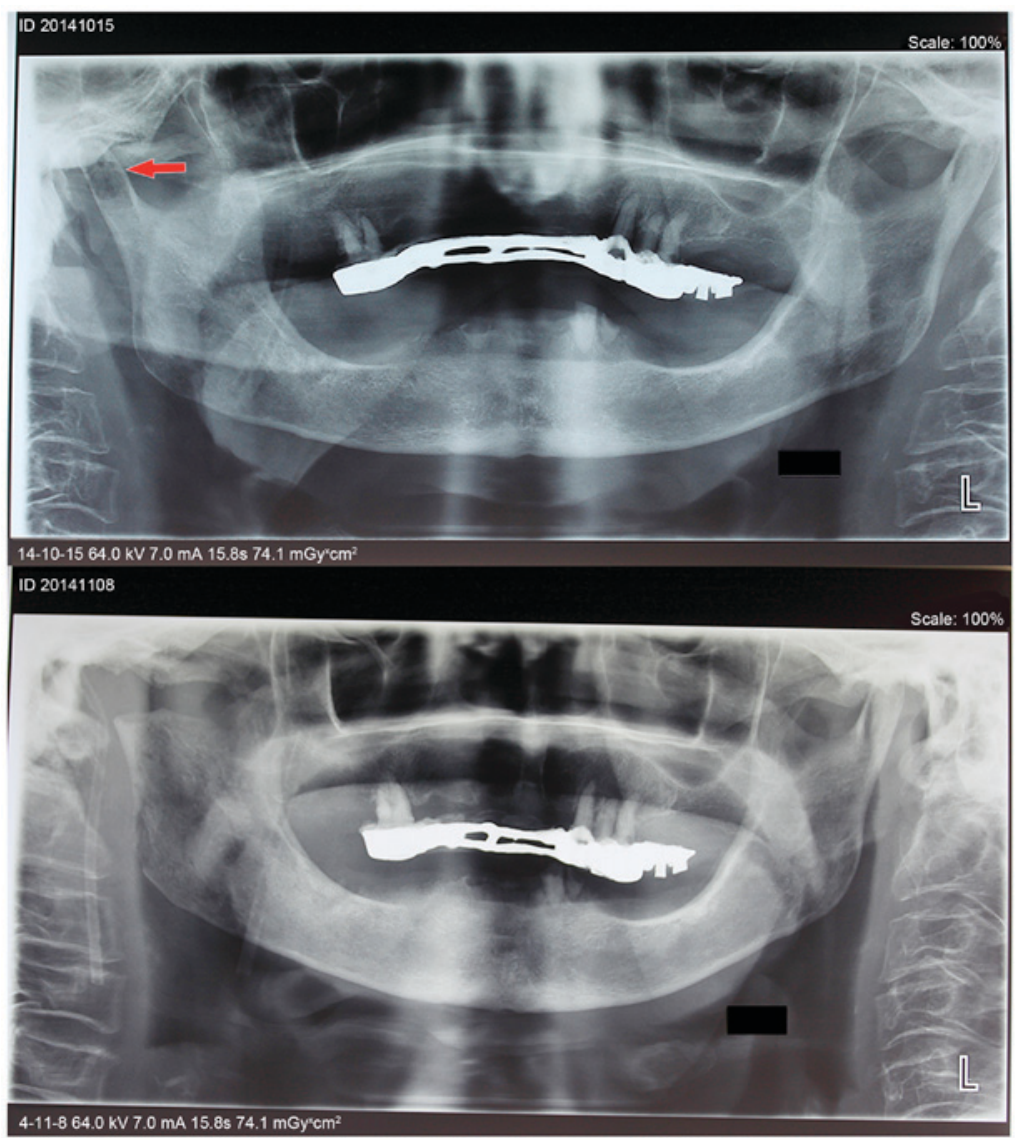

Figure 4. Pre- and post-operative panoramic radiograph findings (upper and lower panel, respectively). The arrow shows that in the pre-operative radiograph, the condylar head was diffusely radiolucent in the middle.

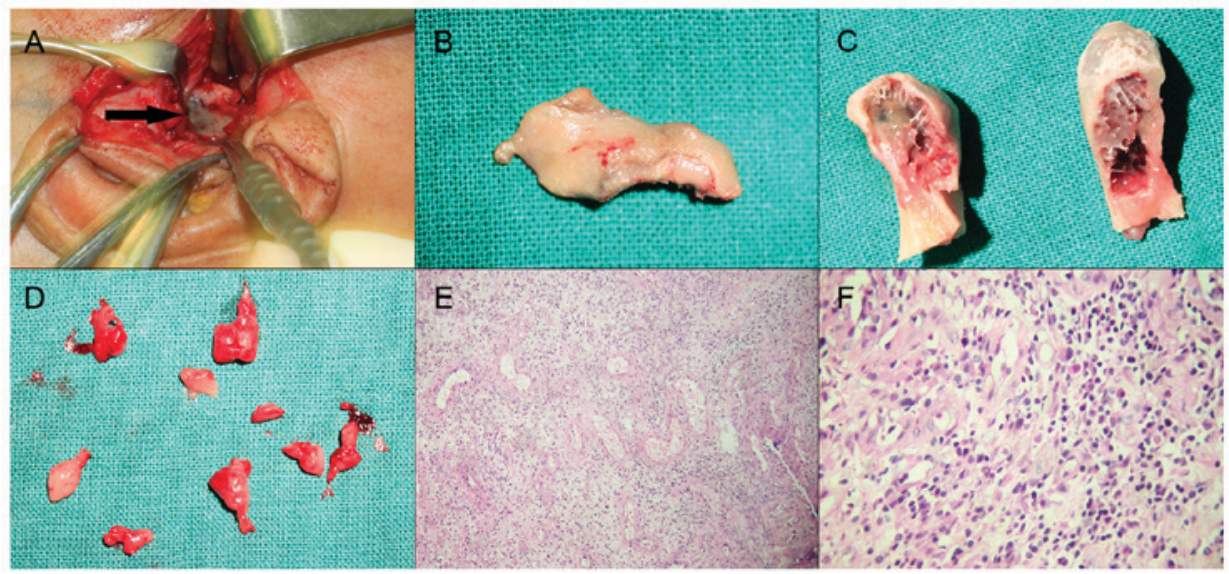

Figure 5. Condylectomy and debridement, specimens affected by SATMJ obtained and histopathologic examination results. (A) Intraoperative image. The arrow indicates a melanized condyle of the right-side mandibule. (B) Metamorphic disk of the TMJ. (C) Necrotic and destroyed mandibular condyle and bone erosion in SATMJ. (D) Inflammatory granulation tissues around the TMJ region after debridement. (E and F) Histopathologic examination showed inflammatory granulation tissue with patchy infiltrates of lymphocytes, magnification of x200 and x400, respectively. SAMTJ, septic arthritis of the temporomandibular joint.

laboratory chemical and microbiological tests as diagnostic approaches (5,26-29). During the clinical examination, an ery thematous and a warm swelling in the pre-auricular region, which is frequently associated with pain, malocclusion, limited maximum mouth opening and mandibular deviation were common symptoms of SATMJ, which were required to be identified, with differential diagnoses being dental abscess or periapical dental abscess, bacterial or viral pharyngitis, retropharyngeal abscess, peritonsillar abscess, acute otitis media, mastoiditis, parotitis, submandibular sialadenitis and lymphadenopathy $(17,29,30)$. In addition, when pre-auricular swelling occurs without obvious signs of inflammation, SATMJ must be considered after excluding cellulitis of the overlying soft tissues, synovial cysts of the TMJ, parotid cysts 
and tumours, condylar hypertrophy and various neoplastic processes arising in the mandibular condyle (31-33). Furthermore, SATMJ must also be distinguished from certain systematic diseases, which may present with similar clinical features, such as rheumatoid arthritis, gout, pseudogout, rheumatic fever and trauma (21,25,34-37). Early diagnosis may not be easy as not all infected TMJs exhibit signs of acute inflammation. Particularly in elderly and immunosuppressed patients, the inflammatory response of SATMJ may be muted (10). In the present case, the signs of inflammation were moderated by fascial attachments surrounding the TMJ until the fistula had formed in the right cheek. As SATMJ was concealed by the abscess, which increased the difficulty to establish the diagnosis, the patient's treatment was delayed. In such circumstances, supplementary examinations were required to confirm the diagnosis, as available findings were not particularly significant.

Once SATMJ is suspected, joint aspiration is recommended to confirm it. The aspirated fluid and turbidity are required to be grossly examined for color as well as subjected to Gram staining, culture and sensitivity analysis $(29,38)$. Owing to their relatively high accuracy and the high rate of the occurrence of bacteria in the synovial fluid, cultures are considered to be a credible method to definitively diagnose SATMJ, but not gonococcal arthritis. Fasting synovial glucose estimation is another form of evidence of infection, which is decreased to less than half the serum value in most bacterial infections and increased in gonococcal arthritis $(10,29)$.

Radiological findings, such as panoramic radiographs, $\mathrm{CT}$ and magnetic resonance imaging (MRI) were able to distinguish between intra- and extra-articular causes of pre-auricular swelling and to evaluate mandibular osteomyelitis and deformity of the TMJ. A plain radiograph may reveal small areas of joint damage obscured. A CT or MRI scan is highly sensitive to early alterations and is useful to demonstrate widening of the articular spaces, bone erosion, effusion and abscess formation in the stage of TMJ infection. It is worth noting that at a time-point at which bone abnormalities are radiographically detectable, accumulation of inflammatory exudate and pus in the TMJ space may have persisted for several weeks, and a CT and MRI scan is therefore advantageous in the diagnosis of patients in the chronic stage $(25,30,39)$.

Laboratory investigations are also helpful for the diagnosis of SATMJ. Increased serum leukocyte and neutrophil counts prompt infections; however, they may be normal in patients treated with antibiotics. C-reactive protein, as a non-specific indicator of inflammation, may be elevated in the initial stage $(5,26)$. Besides, arthroscopy is advocated as an aid for diagnosing SATMJ and a negative technetium 99 scan of the joint can rule out the diagnosis of arthritis $(10,27)$.

At present, no clear consensus on the management of SATMJ has been reached, and thus, different methods have been proposed for joint drainage and decompression in various SATMJ cases, including needle aspiration, arthroscopy and arthrotomy (40). To keep potential therapy-induced risks of the TMJ to a minimum, needle aspiration was often selected as an initial way to relieve the pain and clear the inflammatory medium; particularly arthrocentesis with double needles was advocated as more effective and irrigation may be performed simultaneously. During needle aspiration, appropriate pressure is suggested to avoid the spread of inflammation. For patients with a mild-to-moderate complaint, arthroscopy can be applied to remove fibrous adhesion and smoothen the articular surface $(5,13)$. However, as soon as the abscess is established, exploration of the TMJ is necessary and surgical drainage must be performed immediately, as the proximity of the TMJ to the skull base facilitates the potential intracranial spread of infection $(6,10,17,41)$. If severe erosion and destruction of the cartilage and bone is identified, condylectomy and joint replacement will be required $(5,19,34)$. Similarly, in the present case, purulent mandibular condyle and TMJ disc were excised, and granulation tissues filled within the joint space were debrided. Of note, a previous study demonstrated that there was no significant difference between surgical drainage and needle aspiration, and based on this viewpoint, it is suggested that SATMJ of pediatric patients should be treated by aspiration alone and washout in the acute setting $(10,29,42)$.

An increasing body of evidence suggested that the detection of pathogens by microbiological culture is important for selecting a suitable and efficient antibiotic therapy $(5,10,13$, $27,29,30,38,40)$. Although empiric antibiotic therapy may not be effective against certain pathogens, systemic antibiotics should be applied if a provisional diagnosis of SATMJ has been made, and a negative result of definitive bacterial culture should not delay early vigorous treatment. Therefore, empiric antibiotics should be given until the pathogenic examinations are performed. The antibiotic treatment regime should be modified according to the culture and the sensitivity results, if they are available $(5,10,13,27,29,30,38,40)$. It is generally advised that antibiotic therapy should be vigorous and the length of treatment should be at least 30 days $(10,34)$. It is worth emphasizing that whatever type of antibiotic, the dosage and length of course selected should be appropriate for the individual patient. When systemic antimicrobials reach adequate concentrations in normal joints, intra-articular irrigation with antibiotics is controversial and may lead a concomitant risk of introducing new microbes into the infected joint $(30,34)$. In the present case, the patient showed a poor response to antimicrobial treatment alone prior to hospitalization, and it was therefore suggested that antibiotics may only be used as an adjunct therapeutic method, while the treatment of severe SATMJ requires removal of the inflammatory lesions.

Joint immobilization is also helpful in the treatment of SATMJ, and once the joint pain subsides and the acute infectious process improves, physical therapy is necessary to prevent the long-term limitation of mouth opening caused by fibrosis and ankyloses of TMJ $(5,10,15-17,26,43)$.

In conclusion, SATMJ may at times not have any obvious early symptoms and its diagnosis is often delayed until reaching an advanced stage. Hence, clinicians should pay more attention to any diagnostic clues for SATMJ, including its clinical manifestations, imaging findings and the results of joint aspiration and joint fluid analysis and cultures. Once the diagnosis of SATMJ is suspected, appropriate therapy must be performed as soon as possible, such as needle aspiration, arthroscopy, arthrotomy and antibiotic treatment, and even condylectomy and joint replacement. Only in this way, an ideal prognosis of SATMJ can be achieved. 


\section{Acknowledgements}

The authors are grateful to Dr Ru Wang from the Department of Stomatology, (The First Affiliated Hospital of Dalian Medical University, Dalian, China) for her kind support and assistance in the preparation of the present study.

\section{References}

1. Lohiya S and Dillon J: Septic arthritis of the temporomandibular joint-unusual presentations. J Oral Maxillofac Surg 74: 87-94, 2016.

2. Gams K and Freeman P: Temporomandibular joint septic arthritis and mandibular osteomyelitis arising from an odontogenic infection: A case report and review of the literature. J Oral Maxillofac Surg 74: 754-763, 2016.

3. Martins J, Almeida S, Nunes P, Prata F, Lobo ML and Marques JG: Grisel syndrome, acute otitis media, and temporo-mandibular reactive arthritis: A rare association. Int J Pediatr Otorhinolaryngol 79: 1370-1373, 2015.

4. Al-Saadi NJ, Bakathir AA, Al-Hashmi AK and Al-Ismaili MI: Temporomandibular joint ankylosis as a complication of neonatal septic arthritis: Report of two cases. Sultan Qaboos Univ Med J 15: e554-e558, 2015.

5. Cai XY, Yang C, Zhang ZY, Qiu WL, Chen MJ and Zhang SY: Septic arthritis of the temporomandibular joint: A retrospective review of 40 cases. J Oral Maxillofac Surg 68: 731-738, 2010.

6. Trimble LD, Schoenaers JA and Stoelinga PJ: Acute suppurative arthritis of the temporomandibular joint in a patient with rheumatoid arthritis. J Maxillofac Surg 11: 92-95, 1983.

7. O'Meara PM and Bartal E: Septic arthritis: Process, etiology, treatment outcome. A literature review. Orthopedics 11: 623-628, 1988

8. Thomson HG: Septic arthritis of the temporomandibular joint complicating otitis externa. J Laryngol Otol 103: 319-321, 1989.

9. Borenstein DG and Simon GL: Hemophilus influenzae septic arthritis in adults. A report of four cases and a review of the literature. Medicine (Baltimore) 65: 191-201, 1986.

10. Bounds GA, Hopkins R and Sugar A: Septic arthritis of the temporo-mandibular joint-a problematic diagnosis. Br J Oral Maxillofac Surg 25: 61-67, 1987.

11. Hilbert L, Peters WJ and Tepperman PS: Temporomandibular joint destruction after a burn. Burns Incl Therm Inj 10: 214-216, 1984.

12. McCain JP, Zabiegalski NA and Levine RL: Joint infection as a complication of temporomandibular joint arthroscopy: A case report. J Oral Maxillofac Surg 51: 1389-1392, 1993.

13. Moses JJ, Lange CR and Arredondo A: Septic arthritis of the temporomandibular joint after the removal of third molars. J Oral Maxillofac Surg 56: 510-512, 1998.

14. Scroggins SE, Baran AS, Angel MF and Caloss R: Distraction osteogenesis as a treatment for retrognathia and obstructive sleep apnea resulting from temporomandibular joint septic arthritis: A case report. J Oral Maxillofac Surg 70: e509-e515, 2012.

15. Sembronio S, Albiero AM, Robiony M, Costa F, Toro C and Politi M: Septic arthritis of the temporomandibular joint successfully treated with arthroscopic lysis and lavage: Case report and review of the literature. Oral Surg Oral Med Oral Pathol Oral Radiol Endod 103: el-e6, 2007.

16. Cai XY, Yang C, Zhang ZY, Qiu WL, Ha Q and Zhu M: A murine model for septic arthritis of the temporomandibular joint. J Oral Maxillofac Surg 66: 864-869, 2008.

17. Leighty SM, Spach DH, Myall RW and Burns JL: Septic arthritis of the temporomandibular joint: Review of the literature and report of two cases in children. Int J Oral Maxillofac Surg 22: 292-297, 1993

18. Mayne JG and Hatch GS: Arthritis of the temporomandibular joint. J Am Dent Assoc 79: 125-130, 1969.

19. Varghese L, Chacko R, Varghese GM and Job A: Septic arthritis of the temporomandibular joint caused by Aspergillus flavus infection as a complication of otitis externa. Ear Nose Throat J 94: E24-E26, 2015.

20. Regev E, Koplewitz BZ, Nitzan DW and Bar-Ziv J: Ankylosis of the temporomandibular joint as a sequela of septic arthritis and neonatal sepsis. Pediatr Infect Dis J 22: 99-101, 2003.
21. Goldschmidt MJ, Butterfield KJ, Goracy ES and Goldberg MH: Streptococcal infection of the temporomandibular joint of hematogenous origin: A case report and contemporary therapy. J Oral Maxillofac Surg 60: 1347-1353, 2002.

22. Studahl M, Bergman B, Kälebo P and Lindberg J: Septic arthritis of the knee: A 10-year review and long-term follow-up using a new scoring system. Scand J Infect Dis 26: 85-93, 1994.

23. Deesomchok U and Tumrasvin T: Clinical study of culture-proven cases of non-gonococcal arthritis. J Med Assoc Thai 73: 615-623, 1990.

24. Esterhai JL Jr and Gelb I: Adult septic arthritis. Orthop Clin North Am 22: 503-514, 1991.

25. Kito S, Hirashima S, Yoshioka I, Habu M, Kodama M, Kokuryo S, Oda M, Tanaka T, Wakasugi-Sato N, Matsumoto-Takeda S, et al: A case of chronic infectious arthritis of the temporomandibular joint associated with osteomyelitis without malocclusion. Open Dent J 4: 29-32, 2010.

26. Klüppel LE, Bernabé FB, Primo BT, Stringhini DJ, da Costa DJ, Rebellato NL and Müller PR: Septic arthritis of the temporomandibular joint. J Craniofac Surg 23: 1752-1754, 2012.

27. Hincapie JW, Tobon D and Diaz-Reyes GA: Septic arthritis of the temporomandibular joint. Otolaryngol Head Neck Surg 121: 836-837, 1999.

28. Cai XY, Yang C, Chen MJ, Zhang SY and Yun B: Arthroscopic management of septic arthritis of temporomandibular joint. Oral Surg Oral Med Oral Pathol Oral Radiol Endod 109: 24-30, 2010.

29. Gayle EA, Young SM, McKenna SJ and McNaughton CD: Septic arthritis of the temporomandibular joint: Case reports and review of the literature. J Emerg Med 45: 674-678, 2013.

30. Chuk R, Arvier J, Laing B and Coman D: Septic arthritis of the temporomandibular joint in an infant. Clin Pract 5: 736, 2015.

31. Janecka IP and Conley JJ: Synovial cyst of temporo-mandibular joint imitating a parotid tumour (A case report). J Maxillofac Surg 6: 154-156, 1978 .

32. Ethell AT: A rare 'parotid tumour'. J Laryngol Otol 93: 741-754, 1979.

33. Papavasiliou A, Sawyer R, Lund V and Michaels L: Benign conditions of the temporomandibular joint: A diagnostic dilemma. $\mathrm{Br}$ J Oral Surg 21: 222-228, 1983.

34. Hekkenberg RJ, Piedade L, Mock D, Baker G and Freeman JL: Septic arthritis of the temporomandibular joint. Otolaryngol Head Neck Surg 120: 780-782, 1999.

35. Li-Yu J, Schumacher HR Jr and Gratwick G: Invasive tophaceous pseudogout in the temporomandibular joint: Misdiagnosis as tumor: Case report and review of the literature. J Clin Rheumatol 6: 272-277, 2000.

36. Nakagawa Y, Ishii H, Shimoda S and Ishibashi K: Pseudogout of the temporomandibular joint. A case report. Int J Oral Maxillofac Surg 28: 26-28, 1999.

37. Copeland M and Douglas B: Ganglions of the temporomandibular joint: Case report and review of literature. Plast Reconstr Surg 81: 775-776, 1988.

38. Al-Khalisy HM, Nikiforov I, Mansoora Q, Goldman J and Cheriyath P: Septic arthritis in the temporomandibular joint. N Am J Med Sci 7: 480-482, 2015.

39. Murakami K, Matsumoto K and Iizuka T: Suppurative arthritis of the temporomandibular joint. Report of a case with special reference to arthroscopic observations. J Maxillofac Surg 12: 41-45, 1984.

40. Bast F, Collier S, Chadha P and Collier J: Septic arthritis of the temporomandibular joint as a complication of acute otitis media in a child: A rare case and the importance of real-time PCR for diagnosis. Int J Pediatr Otorhinolaryngol 79: 1942-1954, 2015.

41. Rimoin DL and Wennberg JE: Acute septic arthritis complicating chronic rheumatoid arthritis. JAMA 196: 617-621, 1966.

42. Goldenberg DL, Brandt KD, Cohen AS and Cathcart ES: Treatment of septic arthritis: Comparison of needle aspiration and surgery as initial modes of joint drainage. Arthritis Rheum 18: 83-90, 1975.

43. Nade S: Acute septic arthritis in infancy and childhood. J Bone Joint Surg Br 65: 234-241, 1983. 